Voix et Images

voixetimages

\title{
L’intertextualité dans les écrits féminins d'inspiration féministe
}

\section{Evelyn Voldeng}

Volume 7, numéro 3, printemps 1982

Anne Hébert

URI : https://id.erudit.org/iderudit/200346ar

DOI : https://doi.org/10.7202/200346ar

Aller au sommaire du numéro

Éditeur(s)

Les Presses de l'Université du Québec

ISSN

0318-9201 (imprimé)

1705-933X (numérique)

Découvrir la revue

Citer cet article

Voldeng, E. (1982). L'intertextualité dans les écrits féminins d'inspiration féministe. Voix et Images, 7(3), 523-530. https://doi.org/10.7202/200346ar d'utilisation que vous pouvez consulter en ligne.

https://apropos.erudit.org/fr/usagers/politique-dutilisation/ 


\title{
L'intertextualité dans les écrits féminins d'inspiration féministe
}

\author{
par Evelyn Voldeng, Université Carleton
}

Étudier la démarche intertextuelle dans les textes de différents auteurs féminins nécessite, à la fois la sélection d'un corpus d'écrits féminins et la délimitation de l'objet théorique conçu et signifié sous le nom d'intertextualité. C'est à partir de la définition même de l'intertexte et des idéologies inter. textuelles que nous avons été amenée à choisir comme corpus, des textes de femmes d'inspiration féministe ', produits dans les années soixante-dix, principalement en France et au Québec, textes se réclamant tous, à divers degrés, de la démarche intertextuelle. Notre travail a consisté à dégager les modalités selon lesquelles, dans la filiation intertextuelle, ces textes s'articulent entre eux, d'une part, et sur d'autres textes du passé et du présent d'autre part, ce qui revient à analyser, en particulier, ces modes et fonctionnements de l'intertextualité que sont le plagiat, le collage, la référence et la parodie.

Le concept d'intertextualité, lancé par Mikhaill Bakhtine occupe une place importante dans la théorie littéraire actuelle, que ce soit Julia Kristeva, Tzvetan Todorov, Michel Butor ou Roland Barthes, tous ont contribué à définir cette notion. Posant avec Julia Kristeva que « tout texte se construit comme mosaique de citations, tout texte est absorption et transformation d'un autre texte ${ }^{2}$. nous définirons l'intertextualité comme le dialogue d'un texte avec tous les textes, avec l'histoire elle-même considérée comme un texte. Les théoriciens distinguant souvent entre une intertextualité générale ${ }^{3}$ (rapports intertextueis entre textes d'auteurs différents), une intertextualité restreinte (rapports intertextuels entre textes du même auteur), une intertextualité externe (rapport $d^{\prime} u n$ texte à un autre texte) et une intertextualité interne (rapport d'un texte à lui-même), une étude de l'intertextualité ne peut nécessairement que porter sur quelques aspects de cette intertextualité. Aussi nous bornerons-nous à analyser, premièrement, les rapports intertextuels entre des textes d'auteurs féminins différents, deuxièmement vis-à-vis des textes de la tradition et de leur arrière-plan idéologique.

Si la problématique intertextuelle est étroitement reliée aux écrits d'inspiration féministe des années soixante-dix, ceci est dû à deux raisons 
majeures: d'une part, la place essentielle accordée aux préoccupations formelles par des femmes-écrivains telles que Madeleine Gagnon, Nicole Brossard, Hélène Cixous, France Théorêt, Germaine Beaulieu ... etc., de l'autre. le rôle de support idéologique que joue dans leurs écrits l'intertextualité.

Quand Hélène Cixous déclare - et ses déclarations sont assumées par les "écrivaines» québécoises - qu'un «corps textuel féminin, c'est toujours sans fin. C'est sans but et sans bout, ca ne se termine pas... Ce qui se passe, c'est une circulation infinie du désir d'un corps à l'autre" ${ }^{4}$, quand Éliane Azoulay, dans le même dossier intitulé "Des Fermmes en écriture» affirme: * Il n'y a pas une femme mais des femmes. Quand elle écrit, elle se cherche en elle-même en tant que d'autres femmes»5, elles insistent sur la continuité qui unit les textes entre eux. Un texte s'élabore à partir de ceux qui l'ont précédé et de ceux parmi lesquels il prend place. Il existe une relation nécessaire d'une écriture à une autre écriture qui la précède ou qui s'inscrit dans le mème horizon temporel. Partant des prémisses qui. stıpulent que toute ceuvre est inachevée et demande d'être poursuivie, que tout texte est pré-texte, la femme écrivain va même jusqu'à faire du discours entre textes qu'est la critique, un discours intertextuel. On peut prendre, comme exemple, les comptes rendus de textes de femmes qui se présentent sous forme de critıque-écriture ou pour reprendre l'expression de Suzanne Lamy de "voyage autour d'une écriture $\infty$.

Tout texte étant produit en connexion avec une matière première fournie par les autres textes et étant destiné à s'insérer dans un con-texte est nécessairement le point d'articulation de divers discours, manifestations idéologiques du langage, ce qui pose d'emblée le problème du support idéologique de l'usage intertextuel. Et s'interroger sur le rôle de l'intertextualité dans les textes de femmes-écrivains comme Madeleine Gagnon, Denise Boucher, Louky Bersianik... permet de mieux cerner le genre de travail intertextuel qui se fait dans ces textes de femmes.

En accord avec le principe que toute œuvre est une œuvre collective, les aécrivaines» renoncent tout d'abord au système de "la propriété et de l'inviolabilité littéraire ${ }^{7}$ ce qui ouvre le champ libre dans leurs écrits à toutes les formes de la citation, en particulier au plagiat et à la variante du plagiat qu'est le collage. C'est ce que Madeleine Gagnon, parmi d'autres, revendique dans la lettre-préface au livre de Denise Boucher Cyprine ${ }^{8}$. "Tes mots détendent mes paroles. Nous allons le leur dire quelle ressemblance nous habite. Comment nous nous répétons, nous plagions; comment nos écritures deviennent collectives." 9 Si la démarche intertextuelle apparaît, d'une part, comme une réactivation du sens, comme un questionnement ininterrompu du corps textuel collectif, l'intertextualité peut fonctionner, d'autre part, comme détournement culturel, voire comme gage d'une subversion politique. C'est en utilisant deux formes particulières de l'intertextualité, la parodie et le pastiche, que plusieurs textes de femmes dénoncent les discours "sexistes " stéréotypés. L'intertextualité peut mème aller si loin qu'elle devient " stratégie du brouillage " 
et s'étend à « tout le discours social. Certains textes de Yolande Villemaire, de France Théorêt font ainsi “délirer les codes."10 Sans toujours aller jusqu'à ce cas extrême, les textes de femmes font cependant de la démarche intertextuelle un usage esthétique révolutionnaire. Elles réclament dans leurs textes de "retailles" "le droit à une pratique de groupe, pratique exploratoire et subversive.

Une fois établie la présence explicite de l'intertextualité au niveau du contenu formel des textes des “écrivaines». il s'agit d'étudier les modes et fonctionnements de cette intertextualité. Trois éléments entrent en jeu dans la démarche intertextuelle: le texte empruntant (ou texte-support), le texte emprunté et le corpus d'origine d'où est extrait le texte emprunté. ${ }^{12}$ La facon dont s'opère la filiation intertextuélle est fonction du type de texte emprunté et des mécanismes selon lesquels se fait la greffe intertextuelle.

Quand un texte unique est a cité sur le mode de la reproduction on obtient le plagiat » ou une "variante particulière du plagiat, le collage. ${ }^{13}$ Chez nombre de femmes-écrivains, entre autres, Geneviève Amyot, Madeleine Gagnon, Denise Boucher, France Théorêt... le recours avoué ou non à d'autres textes, le démarquage dans le parallélisme de discours complémentaires ou opposés prend souvent la forme de montage, de collage. Denise Boucher dans Cyprine, au sous-titre significatif, "essai-collage pour être une femme». prend, par le jeu intertextuel la parole au nom de toutes les femmes. Dans Cyprine on passe du poème cité au fragment de texte dramatique, de la chanson populaire au fragment d'essai et a la citation didactique. Par ce jeu renouvelé de citations se réfléchissent dans le texte tous les textes des femmes dans une sorte d'intertextualité diachronique et synchronique. Les textes empruntés qui forment le tissu culturel au sein duquel apparait Cyprine sont extraordinaire. ment nombreux et divers allant d'une citation tirée des Misères de la femme mariée de. Nicole Estienne à La nef des sorcières (1976) et à En beau fusil de Francine Déry (1978). Les citations appartiennent aux deux bouts de la chaine du langage. à la culture première ou populaire, comme, par exemple, les vieilles chansons du folklore, les légendes, ou à la culture seconde, au discours savant comme celui de Julia Kristeva, entre autres. Dans l'essai-collage de Denise Boucher le problème de l'intertextualité a été de faire tenir plusieurs textes. et même des systèmes signifiants de type figuratif, en un seul texte, sans qu'ils se détruisent les uns les autres, ni que l'intertexte ne se désagrège en tant que totalité structurée. Nombreux textes de femmes que ce soit Retailles, ou Journal de l'année passée ${ }^{14}$ par exemple ont systématiquement fait éclater le cadre narratif selon certaines techniques de déconstruction. Par une utilisation systématique du collage et du montage on voit apparaitre de nouveaux textes hybrides dont le but conscient est de revaloriser certains genres «mineurs»: le reportage, le journal intime, le roman épistolier... Dans Cyprine dont le titre même est emprunté aux Guérillères ${ }^{16}$, livre de l'«écrivaine» Monique Wittig, le corps textuel est encadré par des lettres de féministes contemporaines. De façon générale, dans le collage. l'enchâssement du fragment textuel peut se faire par des liens syntaxiques. Dans d'autres cas, il peut 
se faire au niveau sémantique, à plus forte raison si l'emprunt est de type figuratif.

La signification à donner au « texte-greffon » dépendra du corpus d'origine du texte emprunté. Si ce dernier appartient aux textes revendiqués par la tradition féministe (textes produits dans leur grande majorité par des femmes) l'intertexte devient, selon les mots de Madeleine Gagnon "cette multitude de textes amants qui d'un siècle à l'autre parfois se rencontrent, sans quoi notre histoire ne serait pas venue. Les citations ne seront donc toujours pour moi que des entrelacements privilégiés où les mots se marquent d'étreintes pour poursuivre ce qui de l'histoire me dit le durable et l'intime. ${ }^{16}$

D'une façon générale, quand un fragment textuel se greffe sur un autre texte, nous avons une réactivation du sens qui peut aller jusqu'à la subversion de la signification élémentaire. Si, à titre d'exemple, on considère dans l'essaicollage de Denise Boucher les fragments textuels empruntés à des textes d'hommes, on voit que les citations tirées de la lettre du voyant de Rimbaud ou de La Sorcière de Michelet s'articulent au texte-support dans une sorte de continuité sémantique. Dans la plupart des cas cependant, la signification de la citation se voit détournée, subvertie par le contexte. La figure de l'intertextualité mise en jeu est alors l'interversion. Elle peut être par exemple interversion de la situation énonciative ainsi quand Denise Boucher, après avoir cité ce fragment de «La Chanson du Mal-Aimé» de Guillaume Apollinaire:

Mais en vérité je l'attends

De tout (sic) mon coeur, de toute (sic) mon âme

Et sur le pont des Reviens-t'en

$\mathrm{Si}$ jamais elle (sic) revient cette femme

Je lui dirai je (sic) suis content,

enchaîne: «Je lui dirai je suis contente à cette femme du bout du monde. Cette femme initiale dont je cherche la trace. 17 La figure de l'interversion correspond au plan textuel à la revendication féministe d'une histoire, d'une culture où tout se trouve inversé; où. comme nous dit Denise Boucher c'est Ronsard qui plagie Louise Labé. ${ }^{18}$

Après avoir considéré la citation d'un texte unique. il convient d'étudier le fonctionnement intertextuel quand les éléments empruntés se réfèrent à l'ensemble des textes du contexte. Nous avons alors affaire à des stéréotypes. discours figés, topoi, clichés et lieux communs. Les styles éculés et les situations types présents dans les livres, "les journaux, la publicité, les messages politiques, le langage de la rue (...) tout ce qui est manifestation écrite et orale collective ${ }^{19}$ se retrouvent dans les textes de femmes comme dans n'importe quel texte. Mais s'y ajoutent des stéréotypes qui se réfèrent au corpus de textes féministes et à l'ensemble des valeurs véhiculées par ces textes. Une sousculture, avec son registre de discours féministe ( “l'ordre des castrants», «l'érotisme phalloçrate» etc.), sa production scripturale, ses "guérillères» comme Monique Wittig appelle ces nouvelles amazones, ses slogans, son 
signe de ralliement, ses chants de combat, s'est en effet développée dans plusieurs pays. Dans ces textes de femmes, reviennent sans cesse certains motifs fondamentaux comme ceux du corps, du sexe, de la matière et de l'écriture, des schémas mythologiques (les Amazones. l'androgyne) mais aussi des clichés, des lieux communs, des fragments textuels, schématisés, sclérosés à force d'être repris d'un texte d'inspiration féministe à l'autre. Les mêmes textes cités (A Room of one's own, Le Deuxième sexe, Nouvelles Lettres portugaises. Les Pérégrinations d'une paria... ${ }^{20}$ ) reviennent sans cesse sous la plume des aécrivaines». Cette stéréotypie se produit d'ailleurs à un rythme d'autant plus accéléré que se développent aes instances de production et de diffusion qui s'adressent principalement aux femmes.

Les discours figés, les stéréotypes sont souvent cités sur le mode du renvoi, devenant ainsi une référence ou une allusion. Les éléments auxquels il est fait référence apparaissent dissimulés dans la substance de l'écrit; ils ne se signalent plus que par des sortes de repères d'indices 'mythologiques' ou 'rhétoriques'. 21 Dans le jeu intertextuel, le recours a l'allusion est particulièrement fréquent chez les femmes-écrivains. C'est en effet par l'allusion. genre « style initié », que s'établit la connivence entre l'auteur et sa lectrice/son lecteur féministe. L'allusion une fois décryptée établit l'adéquation entre les intentions de l'auteur et l'horizon d'attente (Jauss) d'un certain public. Le signifiant «sorcière" par exemple apparaissant dans une cuvre féministe renvoie à toute une suite de textes dont la matrice est vraisemblablement La Sorcière de Michelet. Dans le cas du schème "sorcière nous assistons même à une interversion des valeurs symboliques. Comme le dit Naomi Goldenberg dans son livre Changing of the Gods (Boston: Beacon Press, 1979): aehind every witch, dragoness and temptress there is a vision of female power." (p. 74).

Dans le collage, le jeu intertextuel peut se faire sur le mode de la subversion. Une autre démarche intertextuelle dont le but est de modifier la signification, la portée et la fonction d'autres textes est la parodie. On obtient la parodie quand on cite un texte unique, l'ensemble des textes d'un écrivain, un genre, une école ou un courant de pensée sur le mode de la caricature. ${ }^{22}$

II est rare que, dans un texte féministe, le texte parodié ou référent soit un texte unique bien qu'il arrive que la parodie de situations stéréotypées, de figures archétypiques et de lieux communs se fasse dans le cadre parodique d'un texte particulier. Ainsi $\angle$ Euguélionne ${ }^{23}$ de Louky Bersianik est une parodie de $L a$ Bible et de L'Évangile. Ceci est déjà préfiguré par le titre car une interprétation étymologique de celui-ci donne le mot grec a euaggelion» bonne nouvelle, Evangile. Dans Le Pique-nique sur I"Acropole du même auteur le texte que la parodie prend comme objet est Le Banquet de Platon. Le référent parodique peut-ètre l'ensemble des textes les plus représentatifs d'un auteur. Dans Noli $M$ Tangere 24 et dans L'Euguélionne nous avons, par exemple, une parodie du discours freudien. Louky Bersianik y ridiculise le paternaliste $\propto$ SaintSiegfried alias Freud et ses enseignements sur le "primat du phallus». II arrive 
que le parodié soit un genre ou un sous-genre littéraire. Dans Archaos ou le jardin étincelant de Christiane Rochefort (Paris: Grasset, 1972) nous avons des exemples de parodie du conte de fées, de l'épopée et de la littérature courtoise. De façon générale, les écrivaines, dans lẹurs textes, dénoncent sur le mode parodique les discours figés véhiculant des mythes, stéréotypes et lieux communs sexistes.

Après avoir considéré le type de textes que la parodie prend en charge. il importe de voir comment la parodie marque sa différence vis-à-vis du modèle choisi. La parodie repose sur un principe fondamental, la caricature «laquelle trouve à s'incarner principalement en deux figures rhétoriques classiques qui sont l'hyperbole et l'antiphrase. ${ }^{25}$ C'est par exemple a l'hyperbole qu'a recours Louky Bersianik quand elle transforme le phallus en «obélisque transcendantal. .

Les procédés parodiques utilisés dans les textes des femmes écrivains visent d'une part "la fiction c'est-à-dire l'univers figuré par le texte o et d'autre part « la narration c'est-à-dire l'ensemble des opérations textuelles qui contribuent à instituer le texte en tant que tel et des conventions qui en règlent le déroulement discursif. 26

Le principal procédé mis en jeu dans la parodie de la fiction est l'inversion. Tantôt c'est la qualification, la situation dramatique ou les valeurs symboliques qui sont inversées. A la généalogie du Christ répond sur le mode parodique celle de l'Euguélionne, troisième personne d'une trinité femelle (mère, fille et cervelle suprême). A l'inverse de ce qui se passe dans la Bible, L'Euguélionne est enfantée par un-père vierge. (p. 53) A la fin de l'immense fresque parodique de Louky Bersianik a le primat du Phallus» est remplacé par son manque, "le primat du Trou». (p. 397)

Au niveau de la narration la démarche parodique utilise des figures jouant sur la morphologie, la sémantique et la syntaxe. Le jeu intertextuel parodique avec ses déformations et distorsions des signifiants et signifiés, de la morphologie, la déconstruction de la syntaxe, est directement lié aux préoccupations formelles des femmes-écrivains. Par «la machine perturbante » de l'intertextualité elles inscrivent “dans la textualité mème et comme objet de cette textualité une problématique de l'écriture.»27 Dans des textes qui empruntent souvent à tous les genres, par l'usage des technicalités de l'écriture en transformation, elles entendent transgresser la forme d'écriture sociale imposée a priori. Ce travail parodique dans et sur le signifiant se veut libération de l'assujettissement du langage à tous les discours figés. Que ce soient des figures portant sur la morphologie: emploi de termes bas, termes de joual, diminutifs (à la fin de L'Euguélionne le pénis n'est plus que ala quéquette à Jésus-Christo), expresșions étrangères, calembours... etc. ou des figures jouant sur la sémantique: déformations de phrases historiques ou de proverbes ( «je me pierre qui roule n'amasse pas mousse», "je suis en bonne et due forme $\times)^{28}$ ou une déconstruction systématique de la syntaxe. toutes ces opérations textuelles participent du même détournement langagier voire culturel. 
Dans leur déconstruction du langage c'est souvent au pastiche qu'auront recours les textes féminins, le pastiche étant la citation d'un texte ou de l'ensemble des textes d'un écrivain sur le mode de l'imitation de style. ${ }^{29}$ Dénoncant la société patriarcale judéo-chrétienne, ces textes prendront souvent comme modèle du pastiche le rituel, les prières et en particulier le * Notre Père *. A Denise Boucher qui s'écrie: “Au nom de la queue, du père et du fils» répond Louky Bersianik: «Au Nom du Trou évacuant. Amen. ${ }^{30}$ Dans certains cas le pastiche peut avoir pour modèle un langage dit masculin, ce langage défini par Francoise Collin comme langage d'auto-représentation, langage autologique, langage opératoire, rhétorique, manipulateur, langage d'érection enfin. ${ }^{31}$

Une étude de l'intertextualité explicitement présente au niveau du contenu formel des textes de femmes d'inspiration féministe, qu'elle prenne l'aspect de la citation, du plagiat, du collage, du montage, de l'imitation ou de la parodie, montre qu'il est difficile de la dissocier de son support idéologique. Que la clé de l'intertextualité soit dans l'histoire du sujet créateur comme le soutient Harold Bloom ou dans l'évolution des média comme le veut'McLuhan ${ }^{32}$ ou probablement dans les deux, il n'en reste pas moins que l'usage intertextuel des discours apparaît de façon massive dans les textes des écrivaines des années soixante-dix. L'intertextualité dans leurs écrits répond à " une vocation critique, ludique et exploratoire.. ${ }^{33}$ Et comme la question de l'écriture est pour elles liée à la problématique de l'élaboration d'une culture nouvelle, il est d'autant moins étonnant qu'elles aient recours à la démarche intertextuelle. «instrument de parole privilégié des époques d'effritement et de renaissance culturels. $n^{34}$

1. Cf. Evelyne Voldeng, "La Poésie contemporaine d'inspiratıon féministe, " Dérives. $n^{\circ} 22,1980$, pp. 3-14.

2. Julia Kristeva, *Bakhtine, le mot, le dialogue et le roman," Critique, avril 1967. pp. 440-441.

3. Lucien Dälenbach, a Intertexte et autotexte, Poétique, n²7, 1976, p. 282.

4. Hélène Cixous. "Textes de l'imprévisible: Grâce à," Les Nouvelles Littéraires, $n^{\circ} 2534,26$ mai 1976, p. 18.

5. Eliane Azoulay, «Et si c'était Nausicaa .... Les Nouvelles Littéraires. Ibid., p. 16.

6. Suzanne Lamy, d'elles, Montréal, L'Hexagone, 1979, p. 53.

7. Claude Bouché, Lautréamont, du lieu commun à la parodie. Paris, Larousse, 1974. p. 15.

8. Denise Boucher, Cyprine essai-collage pour être une femme. Montréal, L'Aurore, 1978.

9. bid., p. 9.

10. Laurent Jenny, "La stratégie de la forme," Poétique, $n^{\circ} 27,1976$, p. 281.

11. Cf. Denise Boucher, Madeleine Gagnon, Retailles, Montréal, L'étincelle, 1977.

12. André Topia, «Contrepoints joyciens, ^ Poétique, $n^{\circ} 27,1976, p .353$.

13. Claude Bouché, op. cit., p. 42, 47.

14. Geneviève Amyot, Journal de I'année passé, Montréal, VLB éditeur, 1978.

15. Monique Wittig, Les Guérilleres, Paris, Minuit. 1974. 
16. Madeleine Gagnon, Retailles, op. cit., p. 158.

17. Denise Boucher, Cyprine, op. cit., p. 17.

18. Ibid., p. 86 .

19. Claude Bouché, op. cit., p. 44.

20. Flora Tristan, Les Pérégrinations d"un paria, Paris, 1838.

21. Claude Bouché, op. cit. p. 45.

22. Ibid., p. 48.

23. Louky Bersianik, L'Euguélionne. Montréal, La Presse, 1976.

24. Louky Bersianik, « Noli M Tangere, $\infty$ La Barre du jour, pp. 56-57, 1977.

25. Claude Bouché, op. cit. p. 48.

26. Ibid., p. 49.

27. Ibid., p. 11.

28. Geneviève Amyot, Journal de lannée passée, op., cit., p. 14.

29: Claude Bouché, op. cit. p. 48.

30. Louky Bersianik, L'Euguélionne, op. cit., p. 397

31. Françoise Collin, "Polyglolulssons, " Les Cahiers du Grif, n 12. 1976, pp. 4-5.

32. Cf. Laurent Jenny, « La stratégie de la forme, * Poétique, $n^{\circ} 27,1976$..pp. 258-259.

33. Ibid., p. 281.

34. Ibid., p. 281. 